\title{
Filigrane
}

Écoutes psychanalytiques

\section{Agir la violence. Quelques déterminants de la violence individuelle et de la violence groupale}

\section{Louis Brunet}

Volume 25, numéro 2, 2016

Le sujet de la violence

URI : https://id.erudit.org/iderudit/1039645ar

DOI : https://doi.org/10.7202/1039645ar

Aller au sommaire du numéro

Éditeur(s)

Revue Santé mentale au Québec

ISSN

1192-1412 (imprimé)

1911-4656 (numérique)

Découvrir la revue

Citer cet article

Brunet, L. (2016). Agir la violence. Quelques déterminants de la violence individuelle et de la violence groupale. Filigrane, 25(2), 9-23.

https://doi.org/10.7202/1039645ar
Résumé de l'article

Cet article présente une analyse des déterminants intrapsychiques de la violence. Les profils psychologiques des individus qui " passent à l'acte " paraissent très divers, allant du commun des mortels au criminel endurci jusqu'au fanatique génocidaire. Comment adviennent la violence individuelle et la violence groupale ? Si l'expression pulsionnelle comme décharge, sans adresse, est à considérer, d'autres manifestations violentes témoignent plutôt d'un message potentiel, c'est-à-dire d'une quête envers l'objet qui saura accorder un sens, permettre la symbolisation. Cet article tend à cerner quelques dynamiques intrapsychiques relevées dans différents cas de figure de la violence actuelle, du criminel local au terrorisme à l'échelle mondiale. 


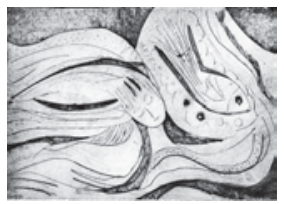

\title{
Agir la violence. Quelques déterminants de la violence individuelle et de la violence groupale
}

\author{
Louis Brunet
}

RÉSUMÉ: Cet article présente une analyse des déterminants intrapsychiques de la violence. Les profils psychologiques des individus qui «passent à l'acte » paraissent très divers, allant du commun des mortels au criminel endurci jusqu'au fanatique génocidaire. Comment adviennent la violence individuelle et la violence groupale? Si l'expression pulsionnelle comme décharge, sans adresse, est à considérer, d'autres manifestations violentes témoignent plutôt d'un message potentiel, c'està-dire d'une quête envers l'objet qui saura accorder un sens, permettre la symbolisation. Cet article tend à cerner quelques dynamiques intrapsychiques relevées dans différents cas de figure de la violence actuelle, du criminel local au terrorisme à l'échelle mondiale.

Mots clés: pulsion, violence, criminalité, violence groupale

e 30 juillet 1932, Albert Einstein écrit à Freud:

Comment est-il possible que la masse se laisse enflammer par lesdits moyens jusqu'à la frénésie et au sacrifice de soi? La réponse ne peut qu'être: En l'homme vit un besoin de haïr et d'anéantir. Cette prédisposition est, en temps ordinaire, présente à l'état latent et elle ne se fait alors jour que chez l'anormal. Mais elle peut être réveillée avec une relative facilité et s'intensifier en psychose de masse. (Freud, 1933, p. 67)

Dans sa longue lettre de septembre 1932, Freud lui répond notamment:

Nous supposons que les pulsions del'homme sont de deux espèces seulement, soit celles qui veulent conserver et réunir - nous les nommons «érotiques» tout à fait dans le sens de l'Eros dans le Banquet de Platon, ou «sexuelles» par une extension consciente du concept populaire de sexualité - et d'autres qui veulent détruire et mettre à mort; nous regroupons celles-ci en pulsion d'agression ou pulsion de destruction. (Freud, 1933, p. 75) 
La pulsion de mort devient pulsion de destruction en étant retournée, avec l'aide d'organes particuliers, vers l'extérieur, sur les objets. Une part de la pulsion de mort demeure toutefois active à l'intérieur de l'être vivant, et nous avons tenté de faire dériver un grand nombre de phénomènes normaux et pathologiques de cette intériorisation de la pulsion de destruction. Nous avons même commis l'hérésie d'expliquer l'apparition de notre conscience morale par un tel retournement de l'agression vers l'intérieur. (Freud, 1933, p. 77)

Pulsion de destruction donc. Mais à cette pulsion de destruction tournée vers l'extérieur s'opposent à la fois la pulsion de mort tournée vers l'intérieur, même liée par Éros dans un masochisme gardien de vie (Rosenberg), et le retournement vers le sujet de cette agression par l'effet du surmoi qui vise à la fois la répression des satisfactions narcissiques grandioses et celle des satisfactions pulsionnelles (Brunet et Casoni, 2003).

Alors pourquoi la violence? La description concise de Freud montre bien que le criminel en chacun ne fait pas un criminel de chacun! Quelles sont alors les conditions et les déterminants intrapsychiques et interrelationnels de l'expression de la violence? Outre l'expression ponctuelle qui peut être le lot de quiconque selon certaines conditions, on retrouve d'une part des criminels ordinaires qui n'hésitent pas à attaquer, certains même à tuer, ceux qui s'opposent à leurs desseins; et d'autre part des gens qui ressemblent au commun des mortels, mais qui, en raison de l'appartenance à un groupe, ou de leur adhésion à une idéologie, en viennent à commettre des actes d'une violence incompréhensible, comme dans un génocide. Ce texte présente une réflexion sur quelques déterminants intrapsychiques et interpersonnels qui rendent possibles tant la violence individuelle chronique que la violence groupale.

\section{Violence décharge et violence signal}

Que ce soit à partir des idées de Winnicott ou d'André Green, la théorie psychanalytique post-1920 ne peut penser la pulsion sans y inclure un potentiel de message qui s'actualisera selon la réponse donnée par l'objet à cette pulsion. La psychisation de la pulsion dépend en grande partie de la réponse que l'objet lui donnera, ne serait-ce que pour que se développe son appropriation subjective par le sujet; appropriation qui fait souvent défaut chez celui qui, chroniquement, agit sa violence.

Dans de nombreuses histoires de criminels et d'individus violents, il semble que le défaut de réponse adéquate de l'objet à sa pulsion ait condamné 
l'enfant à vivre sa pulsionnalité essentiellement comme une décharge plutôt qu'une satisfaction, l'ait condamné à ne pas pouvoir s'approprier subjectivement sa pulsionnalité et à vivre dans un monde dans lequel la pulsion n'est pas messagère. Non seulement chez l'être chroniquement violent la pulsion n'est-elle pas vécue comme messagère pour l'objet, mais elle ne l'est pas pour le sujet lui-même. Elle ne lui fait pas de sens. À défaut que sa pulsion ait été accueillie comme un message faisant du sens, le criminel ou l'homme violent est condamné à ne pas comprendre le message de sa propre pulsionnalité, à ne pas pouvoir la transformer psychiquement, ce qui le condamne à l'agir pour l'expulser. Que peut faire l'enfant quand il ne trouve pas de réponse adéquate à sa pulsion; lorsque l'absence de réflexivité de l'objet ne lui permet pas de s'approprier sa pulsionnalité? Dans le modèle métapsychologique kleinien, cette inadéquation de la réponse confirme à l'enfant la destructivité de toute pulsion. Sur le plan purement quantitatif et dans une optique un peu plus bionnienne ou même winnicottienne, l'absence de réponse suffisante de l'objet à la «quantité» pulsionnelle ne permet pas à l'enfant de transformer cette quantité en qualité, ne permet donc pas le développement de représentations pulsionnelles qui pourraient alors être manipulées par le psychisme (d'abord par la symbolisation, puis par le refoulement) et donc laisse peu de solutions qui ne seraient pas de l'ordre de la décharge: décharge agir, décharge expulsion ou décharge projection. L'absence de réponse suffisante de l'objet à la quantité pulsionnelle provoque chez l'enfant un sentiment d'impuissance à maîtriser la pulsion. Alors le moi des jeunes délinquants, des criminels et des hommes violents est souvent dans la position suivante: «Puisque je ne peux maîtriser ce cheval fou, j’irai où il me mènera. À défaut de le maîtriser, j'en tiendrai les guides; je ferai alliance avec lui pour aller où il voudra me mener.»

L'utilisation du terme de "violence» comme le fait Balier (1988) nous semble intéressante dans l'étude des agressions ou des criminels, car ce terme réfère implicitement à la notion d'intensité et donc à l'importance du point de vue économique en psychanalyse. Et justement, les entretiens avec des criminels nous montrent que, souvent, la problématique de l'homme violent tient à son incapacité à "psychiser» sa pulsionnalité, de sorte qu'elle continue à être vécue comme une pure quantité, ce qui lui laisse peu d'autres choix que d'agir quantitativement sur elle. À la fois, on peut y voir l'effet d'une forme de désintrication pulsionnelle (Balier, 1988), mais aussi une incapacité du psychisme à transformer la pulsion pour la symboliser et donc à la manipuler psychiquement. Faute de pouvoir être psychisée, la pulsion 
est elle-même violente et, dans certains cas, l'acte se substituera à la pensée. Ainsi, ce n'est pas tant que l'homme violent n'ait pas de surmoi, mais plutôt que celui-ci d'une part ne peut agir efficacement sur des «quantités» non psychisées et, d'autre part, que l'agressivité libre investissant elle-même le surmoi doit à son tour être combattue, souvent expulsée, laissant ainsi libre cours au règne du Moi idéal (Casoni et Brunet, 2007). En somme, ce n'est pas simplement que la pulsionnalité serait débridée ou animée d'une force irrépressible, c'est que l'appareil psychique ne semble pas avoir développé les capacités à psychiser cette quantité qui fait violence de l'intérieur.

Ce serait une erreur toutefois de croire que tout homme "violent» ou tout criminel chronique serait totalement incapable de psychiser et de s'approprier subjectivement sa pulsionnalité. Nous croyons qu'il faut plutôt concevoir cette question comme un continuum. D'un côté se situent des organisations psychiques supportant si peu les tensions qu'elles doivent être rapidement expulsées par des actes. De l'autre côté du continuum se situent des organisations psychiques pour lesquelles la difficulté de psychisation se vit tout de même dans un contexte relatif de rapport à un objet et même dans une position relationnelle où la pulsion serait un message en attente d'un objet qui saurait lui apporter une réponse adéquate.

La psychanalyse nous a d'abord fait connaitre la pulsion par son exigence de décharge (point de vue économique). Freud décrit le but de la pulsion comme la satisfaction d'un désir "qui ne peut être obtenue qu'en supprimant l'état d'excitation à la source de la pulsion» (Freud, 1915, p. 18). Mais la pulsion est non seulement à la recherche de la satisfaction (pleasure seeking), mais à la recherche d'un objet (object seeking), car le premier ne peut se réaliser sans le deuxième. Et pour unifier ces deux aspects, René Roussillon explique que le rapport du sujet à l'objet est d'abord et avant tout celui de la recherche d'un "plaisir avec l'objet», d'un plaisir partagé. Du reste, en plus de son aspect quantitatif, Freud décrit la fonction de représentation de la pulsion. Ainsi, la pulsion, outre son impératif de décharge, contient un message potentiel pour le sujet ainsi que pour l'objet. Tout comme l'angoisse constitue un signal du moi, la pulsion est aussi un signal qui informe l'appareil psychique et qui constitue un appel envers l'objet. Une des premières fonctions du parent envers son enfant est justement de comprendre et de répondre aux signaux pulsionnels de celui-ci et par le fait même d'être une sorte de miroir symbolisant de la quantité vécue par le bébé. «Tu as faim mon petit glouton...» dit la mère à son nourrisson, l'aidant peu à peu à symboliser et "psychiser» cette quantité violente qui 
l'assaille. Ce «miroir» de l'objet permet le développement de plusieurs fonctions psychiques qui deviendront essentielles dans le maniement pulsionnel, miroir permettant la reconnaissance d'une quantité; d'une quantité qui ne détruit pas l'objet, d'une quantité qui peut être contenue et donc maniée par l'appareil psychique.

Même dans les gestes violents des adultes, on peut déceler à l'occasion l'empreinte d'un message et d'une demande de réponse de la part de l'objet; réponse qui pourrait n'être qu'une fonction miroir, une fonction de satisfaction pulsionnelle, mais aussi quelquefois une fonction contenante (Brunet, 2010). «Que peux-tu faire de cette violence? Peux-tu en faire quelque chose pour moi? Peux-tu y survivre?»; nous pourrions traduire ainsi certaines des demandes inconscientes adressées à l'objet à travers l'expression pulsionnelle. Comme pour le bébé, il arrive que la pulsion ne puisse être correctement psychisée, mais que le sujet espère tout de même confusément une réponse de l'objet à cette quantité difficilement représentable. Cette réponse permettra ou non l'appropriation subjective de la pulsion; c'est la réponse qui fera en sorte que la pulsion sera un message réussi pour le sujet luimême. Dans certains cas, il arrive que l'échec du processus de symbolisation provoque une forme de retournement relationnel, pour lequel la notion d'identification projective semble utile afin d'en comprendre la dimension potentiellement relationnelle. Il s'agit ici du modèle «communicatif» de l'identification projective (Brunet, 2000), qui peut permettre dans certains cas une reprise des capacités de symbolisation figées (Brunet, 2010), grâce à l'espoir qu'une réponse de l'objet à cette identification projective puisse faire renaître ou remettre en marche la symbolisation - donc une identification projective à potentiel symbolisant, à condition qu'un objet puisse apporter une réponse adéquate. Bien que des auteurs comme Kurt Eissler (1950), Redl et Wineman (1951) ainsi que Mailloux (1971) décrivent comment l'agir vient «à la place» d'un ressenti d'angoisse ou de déplaisir; et bien que Balier (1988) décrive une «tendance incoercible à la décharge» (1988, p. 192) suivant laquelle «l'acte se substitue entièrement à la pensée dont les contenus sont particulièrement pauvres» (1988, p. 192), nous préférons situer la problématique agir-décharge sur un continuum relationnel. Dans certains cas, l'agir signe la négation de l'objet comme «autre je», alors que dans d'autres cas, l'objet peut conserver minimalement le statut «d'autre je» et posséder une potentialité objectalisante. Chez certains pédophiles, il semble d'ailleurs que les gestes se situent dans ce vecteur à potentiel relationnel, objectalisant et symboligène. 
André (nom fictif) est un pédophile qui a été rencontré dans le cadre d'une expertise psycholégale. À plusieurs reprises, il attirait de jeunes garçons chez lui et leur faisait des attouchements. André avait vécu une jeunesse difficile, dans une famille où son père était soit absent, soit violent avec lui. La violence de son père n'était pas que physique; celui-ci l'humiliait, le dévalorisait. Il aurait voulu que son fils réussisse dans les sports alors qu'il était gauche et maladroit. Le père ne pouvait s'identifier à ce fils «inadéquat» et le fils ne pouvait s'identifier au projet que lui proposait son père. André était un enfant qui souffrait de solitude. Il était isolé et était le bouc émissaire à l'école. Des entretiens avec lui montrent que celui-ci ne croyait pas faire du mal aux jeunes garçons lors de ces attouchements. Bien qu'une vision extérieure et objective pouvait qualifier ces gestes «d'agression sexuelle» (donc de violence), ce n'était pas la vision d'André, qui n'y voyait plutôt que de la tendresse. Il ne leur demandait rien en retour, disait-il. Il les «aimait». Les entretiens laissent voir que par identification projective, André est lui-même l'enfant sur lequel il fait des attouchements. On pourrait comprendre que le «je» d'André devient l'enfant sur qui il fait des attouchements qualifiés d' «affectueux». André semble rejouer par identification projective une relation père-fils, une relation homosexuelle dans laquelle il s'identifie non pas au père qu'il a eu, mais au père qu'il aurait voulu avoir et aime l'enfant en qui il se projette en tant qu'enfant: le père aime enfin André par identification projective. Seulement voilà, il n'y a pas et il ne peut y avoir de réponse de l'objet qui lui permettrait de remettre en jeu la symbolisation et l'appropriation subjective. L'objet dans ce cas est un enfant qui ne peut remplir le rôle symbolisant qui pourrait faire évoluer l'identification projective.

En somme, certaines violences peuvent se situer du côté d'une décharge, d'un processus davantage quantitatif que qualitatif, mais d'autres violences se situent plutôt du côté d'un "agir à potentiel de message», d'un agir en quête d'objet qui saura en faire du sens, en permettre la symbolisation et la subjectivation.

\section{Du simple criminel à la violence groupale; quelques déterminants}

Au-delà des aléas de la transformation du quantitatif en qualitatif abordés précédemment, l'étude de la violence individuelle simple, typique notamment des criminels, peut laisser voir une série de déterminants intrapsychiques qui semblent jouer un rôle important dans l'agir. Parmi ces déterminants, certains se retrouvent aussi dans la violence groupale, mais à 
condition de concevoir le groupe lui-même comme l'équivalent d'un appareil psychique groupal (Brunet, 2007; Kaes, 1976) et de concevoir aussi que ce groupe puisse se définir virtuellement et subjectivement en cette ère de la communication internet.

Tout en considérant qu'un être violent puisse se situer à un point ou à un autre du continuum «décharge-message» et que ce point ne soit pas fixe, mais puisse se modifier constamment en raison des aléas de la vie et des conditions relationnelles en jeu, certains facteurs dynamiques et structuraux sont pratiquement toujours en cause chez les personnes violentes. Dans le présent texte, nous nous centrerons sur quelques éléments dynamiques communs de la violence individuelle et de masse, soit le renversement de l'importance dynamique du Moi idéal et du surmoi et le traitement fait à l'objet, par le biais du mépris, de la négation de l'importance de l'objet, de la négation de la dépendance et de la désidentification. Ces deux séries de facteurs dynamiques qui sont intimement reliés se retrouvent tant chez les criminels violents que dans le fonctionnement groupal, tel que celui des groupes terroristes ou celui des mouvements de radicalisation.

Les entretiens réalisés par le groupe de recherche sur la violence individuelle et groupale à l'Université du Québec à Montréal montrent comment nombre de criminels et de meurtriers présentent un investissement démesuré du Moi idéal au détriment de l'investissement du surmoi. Il n'est pas question de prétendre de façon réductrice que l'homme violent n'a pas de surmoi ou même qu'il ne présente qu'un surmoi lacunaire, comme l'écrivaient Johnson et Szurek (1952). Ces entretiens montrent que chez les hommes violents et les criminels, l'essentiel de l'investissement se porte sur le moi idéal. Comment, de façon générale, chez tant de criminels et d'hommes violents, pouvons-nous retrouver si systématiquement cette inversion dynamique de ces deux instances?

L'enfance de bien des criminels montre un vécu d'humiliation, de blessures narcissiques, de dévalorisation, d'impuissance, de violence subie. L'être violent adulte ne le laisse voir que très rarement, mais il semble le plus souvent s'être bâti sur le vécu d'un enfant blessé et impuissant. Si d'un côté, comme nous l'avons mentionné précédemment, «l'objet-environnement» n'a pas soutenu le développement d'une symbolisation suffisante et d'une appropriation subjective des pulsions, les blessures narcissiques et l'impuissance semblent avoir nécessité une formation défensive bien particulière, orientée par une forme d'identification à l'agresseur. Comme de nombreux entretiens le montrent, l'enfant humilié, l'enfant impuissant, a organisé une 
forme de retournement du passif en actif, en s'identifiant, dans le moi idéal, à l'agresseur, à celui qui humilie, à celui qui a trahi sa confiance ou l'a abandonné. Qu'il s'agisse d'actes concrets de violence et de rejet ou de traumas relationnels précoces, les futurs êtres violents ne peuvent plus supporter la dépendance à l'objet qui est un risque trop grand d'abandon, d'humiliation et de blessure narcissique. En s'identifiant, dans le moi idéal, avec celui qui les blesse, ils renversent la position souffrante, deviennent les agresseurs (avec en prime l'augmentation du sentiment de grandiosité) et soumettent leurs objets à ce qu'ils craignent le plus.

En somme, l'être violent dira «je suis puissant, je suis mauvais, je suis dangereux. Plus jamais je ne serai humilié, blessé, déçu ou abandonné. C'est moi qui blesserai, qui humilierai. J'aurai le contrôle, même si je me fais tuer. Il vaut mieux être puissant et méchant que bon et impuissant».

On voit bien qu'un tel surinvestissement du moi idéal ne peut se faire qu'au détriment de l'influence du surmoi. L'alliance avec le Moi idéal, s'il permet de se sentir puissant, invulnérable, à l'abri du besoin objectal (risque de souffrance), s'il promet la satisfaction sans limite, ne peut se faire qu'en réduisant l'influence du surmoi, notamment en le projetant. Comme conséquence dynamique, la valorisation personnelle et l'estime de soi ne sont plus liées aux impératifs du surmoi, à l'observance de normes morales individuelles et sociétales, mais à la satisfaction des idéaux grandioses du moi idéal. Il est possible de se sentir valorisé par le mal. Être le «plus grand voleur» ou «être le plus puissant» devient plus important pour l'équilibre narcissique qu'être bon ou que se réaliser dans un domaine moralement acceptable.

\section{Normand}

Normand est un meurtrier rencontré à une vingtaine de reprises pour des entretiens libres dans le cadre d'une recherche universitaire. Il a commis des meurtres, en plus de divers crimes, tout en reconnaissant à la fois les normes sociales (les lois) et les normes morales («il est mal de tuer» et «la famille de celui qui est tué en souffre»). Cependant, Normand explique sa carrière de meurtrier en disant:

Je voulais devenir quelqu'un de célèbre... célèbre oui... J'ai eu mon nom dans Allo Police.

Normand dit aussi: 
Agir la violence. Quelques déterminants de la violence individuelle et de la violence groupale

Ben $[. .$.$] c'est quand même un tabou énorme chez l'être humain de tuer$ quelqu'un [...] et d'avoir franchi ce tabou-là, il y avait des fois [...] "fierté ne serait pas le bon terme, mais [...] un genre de contentement, de réussite. Tu sais, $\mathrm{j}$ 'avais réussi quelque chose que pas beaucoup de gens ont fait [...] J'étais allé loin, [...] j'étais allé plus loin que le commun des mortels [silence], mais j'étais tout à fait conscient, même à l'époque, [que] c'était affreux ça, mais je m'étais autorisé, c'est vaniteux ça là, tu sais, enlever une vie humaine là c'est [petit sifflement] Ouh! C'est quelque chose là.

Normand ne délire pas, il a conscience du bien et du mal, mais son équilibre narcissique ne dépend pas des notions de bien et de mal que reconnaît pourtant le surmoi. Le moi est plutôt en collusion avec le moi idéal grandiose et sa valeur tient d'être "plus fort» que le surmoi, d'être au-dessus des lois, de s'être libéré des contraintes surmoïques alors que le commun des mortels y est soumis. On pourrait presque dire que ce type d'homme violent n'est pas immoral, mais amoral, tellement le surmoi a été dynamiquement éliminé.

Donc on constate une inversion du sentiment d'humiliation par l'identification à l'agresseur dans le moi idéal et un triomphe du moi idéal sur le surmoi qui est relativement évacué, l'étape suivante étant la mise en acte relationnelle de ce triomphe et de cette inversion. Non seulement l'être violent devient puissant et s'autorise toutes les transgressions, mais sa dynamique de renversement fait que maintenant il n'aura de cesse d'humilier et de triompher de ses objets. Le renversement décrit plus haut lui permet de voir et traiter l'objet avec mépris et de remettre en scène le scénario inversé qui a motivé cette organisation défensive. Mépris, contrôle, triomphe, humiliation et négation de la dépendance normale constitueront alors un processus menant à la désidentification (ou à l'absence d'empathie par la négation de l'idée que l'autre est un autre semblable) et à la possibilité de violence extrême.

L'analyse des phénomènes génocidaires est peut-être ce qui met le plus en lumière ce processus de désidentification menant à la possibilité de violence extrême, en l'absence d'inhibition surmoïque et de culpabilité. Dans le livre Une saison de machettes, Jean Hatzfeld (2003) décrit un processus implacable de déshumanisation visant les Tutsis. L'auteur donne la parole à de nombreux Hutus qui ont été acteurs des massacres. Leur discours est tout à fait éloquent en relation aux processus de désidentification et au traitement de l'objet que nous avons décrits. 
D’une part, leurs propos montrent un processus partagé de déshumanisation, de mépris, faisant en sorte que les Tutsis graduellement en sont venus à ne plus avoir le statut d'êtres humains. À un moment s'est généralisée l'utilisation du mot «inyenzi » pour les désigner, mot qu'on peut traduire par «coquerelle» ou «blatte». Quel est l'effet de partager groupalement la représentation d'un autre comme un non humain, comme une coquerelle? Ce partage collectif favorise la désidentification (l'autre n'est plus un autre semblable) et favorise le travail du clivage et de la projection qui permet à son tour une rupture du lien identificatoire avec l'objet. Nous sommes en présence d'un travail de désymbolisation et de désidentification qui permet de vivre ceci:

F. dit: «J'ai cassé la tête d'une maman d'un coup de gourdin. [...] Je n'ai pas ressenti la mort au bout de mon bras. Je suis rentré le soir chez moi sans même y penser.» (Hatzfeld, 2003, p. 25).

Par contre, certains rapportent plutôt des moments où tuer était plus difficile. Ainsi P. dit:

Je me souviens toutefois de la première personne qui m’a regardé, au moment du coup sanglant. Ça, c'était quelque chose. Les yeux de celui qu'on tue sont immortels, s'ils vous font face au moment fatal [...] Les yeux du tué, pour le tueur, sont sa calamité s'il les regarde. Ils sont le blâme de celui qu'il tue. (Hatzfeld, 2003, p. 26)

Si les regards se croisent, il y a donc possibilité du retour de l'humain, à savoir la possibilité de revoir en l'autre, un autre être humain et non pas seulement une coquerelle.

Lorsque la télévision ou des vidéos diffusées sur internet nous montrent des membres du groupe État Islamique décapiter des gens prostrés, mettre le feu à des hommes attachés mais vivants ou noyer des prisonniers enfermés dans une cage, il faut bien imaginer que pour eux aussi, les êtres humains qu'ils massacrent ne sont pas des «autres semblables à eux-mêmes» et qu'un processus de désidentification s'est enclenché, soutenu par le mépris, le clivage et la projection.

\section{La radicalisation chez les jeunes Occidentaux. L'errance identitaire et le chant des sirènes du moi idéal}

À la lumière des analyses du cheminement de certains terroristes (comme les entretiens des Brigades rouges par Carole BeeBe Tarantelli, 2010) ou du 
cheminement de membres de sectes religieuses devenues violentes, il est possible d'extrapoler et d'appliquer certaines hypothèses au développement de la radicalisation et de l'attirance vers les groupes extrémistes chez certains jeunes Occidentaux.

Comme Beebe Tarantelli l'a montré suite à ses entretiens avec des membres des Brigades rouges italiennes, ceux-ci comprenaient tout à fait qu'il était mal de tuer des femmes, des enfants et des innocents lors de leurs attentats, mais ils considéraient que ces conséquences «malheureuses» n'étaient que le prix à payer pour l'avancement de la cause. Certains disaient même qu'ils étaient «bien entendu» malheureux de ces morts innocentes, mais qu'ils referaient tout de même le même geste, selon la même logique. Cette façon de voir illustre un clivage important entre les interdits provenant du surmoi et les idéaux grandioses issus du moi idéal, au sein de la personnalité. En deux mots: ici encore, les interdits du surmoi n'ont pas d'influence inhibitrice devant les idéaux grandioses du moi idéal. Ces derniers peuvent donc être agis malgré tout.

C'est le même type de clivage que l'on retrouve chez les membres de sectes religieuses et dans le discours de jeunes gens qui se radicalisent et tentent de quitter le Québec pour rejoindre le groupe État Islamique ou tout autre formation radicale. L'examen de leur parcours lors de l'adolescence, de leur recherche d'une cause à épouser alors qu'ils sont jeunes adultes, les rares entretiens qu'ils ont donnés ou ce qu'ils ont dit à certains amis laissent croire que ces jeunes radicalisés étaient des individus à la recherche d'un «idéal» allant définir totalement leur identité et effacer leur sentiment d'insuffisance sinon d'insignifiance. Bien sûr, dans une certaine mesure, tous les jeunes adultes sont à la recherche d'une cause, d'un idéal donnant un sens à leur vie. Certains le trouvent dans la politique, d'autres dans des causes environnementales et communautaires, certains investissent passionnément un hobby ou une cause politique, mais tous ne versent pas dans la violence pour autant. Le cheminement de radicalisation montre la conjonction d'une importante errance identitaire, de la quête fondamentale d'une identité d'emprunt qui contre-investira le vide narcissique et le sentiment de futilité et d'une adhésion massive à une cause idéalisée procurant l'illusion d'une identité grandiose. La conjonction de la fragilité narcissique identitaire et de la promesse, souvent manipulatrice, de l'atteinte de la grandiosité semble souvent être la clé d'une série de modifications dynamiques qui mèneront presque inéluctablement à la violence. Une suite logique de conséquences dynamiques s'ensuivra dans la lignée suivante: 
- Errance identitaire et recherche d'une identité à endosser;

- La découverte d'une cause et l'idéalisation de cette cause, du groupe, du leader;

- L'identification à ce nouvel idéal grandiose qui accentue l'investissement du moi idéal - premier aspect du clivage, à savoir le vécu de grandiosité;

- Le surinvestissement du moi idéal provoquant une inversion de l'importance dynamique du surmoi et du moi idéal;

- L'identification-création (par analogie au concept de "trouvécréé») par projection d'un ennemi à combattre sinon à détruire deuxième aspect du clivage. Cet ennemi peut être inconsciemment lié à des objets infantiles (triomphe sur le père notamment);

- L'utilisation d'une série d'attributions psychiques clivées visant à réduire l'humanité de cet ennemi (mépris, triomphe, désengagement identificatoire, désubjectivation, démonisation);

- La désidentification d'avec ces personnes vues comme «le démon» ouvre sur la possibilité de l'acte de tuer sans que le surmoi inhibe cette expression extrême de l'agressivité.

\section{L'hypothèse d'un trauma narcissique - l'humiliation}

Plusieurs auteurs font état d'une enfance traumatique chez les criminels, comprenant de la violence de la part du père, des humiliations, des abandons précoces ou de la négligence. Certains en font le point de départ d'une organisation psychique visant à nier l'importance de la relation normale de dépendance, et renversant le vécu infantile traumatique pour ainsi devenir celui qui contrôle, celui qui est sadique, celui qui humilie (Casoni et Brunet, 2007). L'étude des mouvements terroristes a souvent apporté le même constat d'une d'humiliation collective motivant le recours à la violence. Cette humiliation est-elle toujours réelle? Jones (2006) décrit bien comment la majorité des études du phénomène terroriste décrivent qu'un sentiment d'humiliation collective semble systématiquement présent, du moins dans le discours des terroristes. Quoi qu'il en soit, on voit facilement comment les leaders terroristes, politiques et religieux, arrivent à se servir d'une humiliation collective, prétendue ou réelle, pour justifier leurs gestes et pour attirer des adeptes.

Nous posons l'hypothèse que les jeunes Occidentaux épousant la cause d'un groupe lointain comme le groupe armé État Islamique trouvent dans le discours de l'humiliation vengeresse la «représentation externe» d'un 
trauma narcissique personnel dans l'enfance. Si, comme le prétendent plusieurs (Brunet, 2007; Jones, 2006), celui qui se radicalise au point de commettre des gestes terroristes vise à instaurer le triomphe de son moi idéal sur son surmoi, il est possible d'imaginer que ce surinvestissement du moi idéal soit survenu par nécessité dynamique après une honte réelle importante, une humiliation réelle importante. L'histoire de certains individus que l'on a décrits comme des "loups solitaires» (Lombard, 2012) montre souvent que ceux-ci adoptent un discours en tout point semblable aux hommes violents qui tentent de compenser une enfance d'humiliation par une inversion dynamique de leur sentiment d'impuissance.

Jusqu'ici, peu d'études ont pu rendre compte des enjeux dynamiques qui motivent de jeunes Canadiens à partir pour la Syrie ou à commettre isolément un acte de terrorisme comme celui de Zehaf-Bibeau sur la colline parlementaire à Ottawa en mars 2015 ou par Martin Rouleau en 2014. L'étude des textes ou enregistrements qu'ils ont laissés nous montre l'aboutissement d'un processus dont la source et l'organisation ne pourraient être mises en lumière qu'après des entretiens approfondis avec eux. Leurs déclarations montrent cependant, tout comme l'étude des déclarations de terroristes (Beebe Tarantelli, 2010; Brunet, 2007), un discours fait d'identification à une cause hautement idéalisée. Ils semblent s'identifier massivement à cette cause et se sentir grandis de cette identification. De plus, la cause et les valeurs défendues semblent d'une importance telle qu'ils se sentent complètement justifiés de ne pas tenir compte des lois et de la morale de leur société et ainsi pouvoir commettre des assassinats. Cet idéal auquel ils sont massivement identifiés transcende donc les droits et les lois. Il est facile d'y voir la trace de la participation massive du moi idéal qui a justement comme caractéristique de supplanter la morale du surmoi (Lussier, 2006). Nous pourrions reprendre ici la phrase de Freud (1921) dans «Psychologie des masses et analyse du moi»: "l'individu abandonne son idéal du moi et l'échange contre l'idéal de masse incarné dans le meneur» (Freud, 1921, p. 68), à condition néanmoins de se rappeler que Freud n'a jamais clairement distingué l'idéal du moi, le moi idéal et le surmoi, contrairement à Lussier. Ainsi, quelqu'un comme Zehaf Bibeau, le 22 octobre 2014, tout en sachant qu'il allait commettre un attentat, pouvait se voir comme quelqu'un de bon et de vertueux:

"Nous sommes des bonnes personnes, vertueux et qui croyons en Dieu, en ses Lois et ses prophètes, que la paix et le salut soient sur eux tous » dit-il dans son message enregistré. 
L'errance identitaire, la recherche une identité compensatoire grandiose, le transfert d'une blessure narcissique infantile vers une blessure collective, le surinvestissement du moi idéal, sont alors des ingrédients dangereux qui n'attendent qu'une étincelle pour exposer.

Pour conclure:

Le sujet idéal du règne totalitaire n'est ni le nazi convaincu, ni le communiste convaincu, mais l'homme pour qui la distinction entre fait et fiction et la distinction entre vrai et faux n'existent plus (Hannah Arendt, 1972, p. 224)

\section{Louis Brunet \\ brunet.louis@uqam.ca}

\section{Références}

Arendt, A. (1972). Les origines du totalitarisme, tome 3: Le système totalitaire. Paris: Seuil.

Balier, C. (1988). Psychanalyse des comportements violents. Paris: Puf.

Beebe Tarantelli, C. (2010). The Italian Red Brigades and the structure and dynamics of terrorist groups. The International Journal of Psychoanalysis, 91 (3), 541-560.

Brunet, L. (2000). L'identification projective et la fonction contenante: illusions nécessaires ou délire partagé? Journal de la psychanalyse de l'enfant, 26, 161-192.

Brunet, L. (2007). Violence et appareil psychique groupal. Topique, 99, 87-96.

Brunet, L. (2010). Limites, transferts archaïques et fonctions contenantes. Dans C. Chabert (dir.), Les psychoses. Traité de psychopathologie de l'adulte (éd. p. 133-172). Paris: Dunod.

Brunet, L. et Casoni, D. (2003). Culpabilité, honte et dynamique criminelle. Au sujet des fonctions anti-pulsionnelles et anti-narcissiques du Surmoi. Revue française de psychanalyse, 5, 1561-1565.

Casoni, D. et Brunet, L. (2007). The psychodynamics that lead to violence. Part 1. The case of the chronically violent delinquent. Canadian Journal of Psychoanalysis, 15 (1), 41-55.

Eissler, K.R. (1950). Ego-psychological Implications of the Psychoanalytic Treatment of Delinquency. The Psychoanalytic Study of the Child, 5, 97-121

Freud, S. (1915). Pulsions et destins des pulsions. Métapsychologie (p. 11-44). Paris: Gallimard, 1968.

Freud, S. (1921). Psychologie des masses et analyse du moi. CEuvres complètes (vol. XVI, p. 1-83). Paris: Puf. 1991.

Freud, S. (1933). Pourquoi la guerre? Euvres complètes (vol. XIX, p. 61-81). Paris: Puf, 1995.

Gabrion, F. et Brunet, L. (2014). Une étude des relations significatives d'un ex-détenu: aux sources identificatoires de la conflictualité. Filigrane, 23 (1), 115-135.

Hatzfeld, J. (2003). Une saison de machettes. Paris: Seuil.

Johnson, A.M. et Szurek, S.A. (1952). The genesis of antisocial acting out in children and adults. Psychoanalytic Quarterly, 21, 323-343.

Kaes, R. (1976). L'appareil psychique groupal. Paris: Dunod.

Lombard, M.-A. (2012). Le loup solitaire, cauchemar de toutes les polices. Repéré à http:// www.lefigaro.fr/actualite-france/2012/03/20/01016-20120320ARTFIG00512-le-loupsolitaire-cauchemar-de-toutes-les-polices.php 
Agir la violence. Quelques déterminants de la violence individuelle et de la violence groupale

Lussier, A. (2006). La gloire et la faute. Essai psychanalytique sur le conflit qui oppose narcissisme et culpabilité. Montréal: Presses de l'Université du Québec.

Mailloux, N. (1971). Jeunes sans dialogue. Paris: Fleurus.

Redl, F. et Wineman, D. (1951). Children Who Hate: The Disorganization and Breakdown of Behavior Controls. Glence: Free press. 